\title{
The role of imaging techniques in the diagnostics and monitoring of treatment of Sturge-Weber's syndrome
}

Authors: Justyna Lipińska ${ }^{1}$, Maria Kowalczuk ${ }^{1}$, Marek Kurowski ${ }^{1}$, Łukasz Lipiński ${ }^{1}$, Magdalena Woźniak ${ }^{2}$ (mentor)

${ }^{1}$ Student Scientific Society at the Department of Pediatric Radiology Lublin

${ }^{2}$ The Department od Pediatric Radiology Lublin

DOI: https://doi.org/10.26800/LV-142-supp5-9

\section{Background:}

Sturge-Weber's syndrome (encephalotrigeminal angiomatosis) is a rare, non-hereditary syndrome of congenital abnormalities. It is one of the phakomatoses. The incidence of this syndrome is estimated at 1 in 20,000 - 50,000 live births. The cause of SWS is a somatic mutation in the GNAQ gene. A typical picture of a person suffering from SWS is a child with vascular malformation on the face - a 'port-wine stain' birthmark. Other clinical symptoms include seizures, glaucoma, headache, stroke-like transient neurological deficits, and behavior problems.

\section{Case presentation:}

A 9-month-old infant was admitted to the hospital with congenital facial cutaneous hemangioma due to sepsis. Three days later, status epilepticus occurred, which was lasting 1.5 hours. MRI with contrast was ordered, which showed enhancement of the arachnoid along the gyri in the right occipital area and the expansion of the fluid space in the cerebral area. There were no calcifications in the brain. Six months after admission, a CT scan was performed, which showed characteristic massive subcortical calcifications in the left frontal lobe and "tram-track" calcifications along the gyri of the cortex of the occipital lobe and the posterior part of the left parietal lobe of the brain.

\section{Conclusion:}

Sturge-Weber's syndrome has a rare but typical radiological presentation. Lesions in the form of calcifications may be poorly visible in imaging in neonatal period and infancy.

Keywords: imaging techniques, phakomatoses, Sturge-Weber's syndrome 